\title{
JMSJ Award in 2015
}

The JMSJ Editorial Committee presents the JMSJ Award to outstanding papers published each year. In 2015, the following two papers were awarded.

Shinya KOBAYASHI, Yukinari OTA, Yayoi HARADA, Ayataka EBITA, Masami MORIYA, Hirokatsu ONODA, Kazutoshi ONOGI, Hirotaka KAMAHORI, Chiaki KOBAYASHI, Hirokazu ENDO, Kengo MIYAOKA, and Kiyotoshi TAKAHASHI:

"The JRA-55 Reanalysis: General Specifications and Basic Characteristics." $J$. Meteor. Soc. Japan, Vol. 93, No. 1, 5-48 (2015).

http://dx.doi.org/10.2151/jmsj.2015-001

Masaki SATOH, Yohei YAMADA, Masato SUGI, Chihiro KODAMA, and Akira T. NODA:

"Constraint on Future Change in Global Frequency of Tropical Cyclones due to Global Warming." J. Meteor. Soc. Japan, Vol. 93, No. 4, 489-500 (2015).

http://doi.org/10.2151/jmsj.2015-025

Masaki Satoh

Chief Editor, JMSJ 